\title{
Key concepts for extending the depth of field with high resolution
}

\section{Conceptos medulares para la extensión de la profundidad de campo con alta resolución}

\author{
Jorge Ojeda-Castañeda $\left(1,{ }^{*}, \mathrm{~A}\right)$ and Cristina M. Gómez-Sarabia( $(2, \mathrm{~A})$ \\ 1. Electronics Department, University of Guanajuato, Salamanca 36885, Guanajuato, México. \\ 2. Digital Arts Department, University of Guanajuato, Salamanca 36885, Guanajuato, México. \\ (*) Email: jorge ojedacastaneda@yahoo.com \\ A: miembro de AMO / AMO member \\ Recibido / Received: 10/09/2012. Revisado / Revised: 07/11/2012. Aceptado / Accepted: 08/11/2012. \\ DOI: http://dx.doi.org/10.7149/OPA.45.4.449
}

\begin{abstract}
:
We discuss two key concepts that are useful for extending the depth of field of an optical system. The first concept associates anamorphic processors with the methods that reduce the impact of focus errors, on the optical transfer function. The second concept links Alvarez-Lohmann lenses with tunable masks for controlling the depth of field, without modifying the size of the pupil aperture.
\end{abstract}

Key words: Depth of Field, Optical Transfer Function, Modulation Transfer Function, Anamorphic Processors, Alvarez-Lohmann lenses, Tunable Spatial Filters, Apodizers.

\section{RESUMEN:}

Se discuten dos conceptos medulares para extender la profundidad de campo de un sistema óptico. El primer concepto relaciona a los procesadores anamórficos con los métodos para reducir el impacto de errores de focalización, en la función de transferencia óptica. El segundo concepto vincula los lentes tipo Álvarez-Lohmann con filtros espaciales que controlan la profundidad de campo, sin modificar el tamaño de la pupila.

Palabras clave: Profundidad de Campo, Función de Transferencia Óptica, Función de Transferencia de la Modulación, Procesadores Anamórficos, Lentes del tipo Álvarez-Lohmann, Filtros Espaciales Sintonizables, Apodizadores.

\section{REFERENCIAS Y ENLACES / REFERENCES AND LINKS}

[1]. P. Jacquinot, B. Roizen-Dossier, "Apodization", in E. Wolf, Edt, Prog. Opt. Vol. 3, pp. 29-186. North Holland, Amsterdam (1964).

[2]. J. Ojeda-Castaneda, L. R. Berriel-Valdos, E. Montes, "Bessel annular apodizers: imaging characteristics", Appl. Opt. 26, 2770-2772 (1987).

[3]. M. Mino, Y. Okano, "Improvement in the OTF of a defocused optical system through the use of shaded apertures", Appl. Opt. 10, 2219-2225 (1971).

[4]. G. Haeusler, "A method to increase the depth of focus by two step image processing", Opt. Commun. 6, 38-42 (1972).

[5]. J. Ojeda-Castaneda, A, Noyola-Isgleas, "High focal depth by apodization and digital restoration", Appl. Opt. 27, 2583-2586 (1988).

[6]. J. Ojeda-Castañeda, L. R. Berriel Valdos, E. Montes, "Spatial filter for increasing the depth of focus", Opt. Lett. 10, 520-522 (1985).

[7]. J. Ojeda-Castaneda, P. Andrés, A. Diaz, "Annular apodizers for low sensitivity to defocus and to spherical aberration", Opt. Lett. 11, 487-489 (1986). 
[8]. J. Ojeda-Castaneda, E. Tepichin, A. Pons, "Apodization of annular apertures: Strehl ratio", Appl. Opt. 27, 5140-5145 (1988).

[9]. S. C. Biswas, A. Boivin, "Performance of optimum apodizers in presence of primary coma", Can. J. Phys. 57, 1388-1396 (1979).

[10]. J. Ojeda-Castañeda, E. Yépez-Vidal, E. García-Almanza, “Complex amplitude filters for extended depth of field", Photonics Lett. Poland 2, 162-164 (2010).

[11]. L. N. Hazra, A. Guha, "Far-field diffraction properties of radial Walsh filters", J. Opt. Soc. Am. A 3, 843846 (1986).

[12]. E. R. Dowski, T. W. Cathey, "Extended depth of field through wave-front coding", Appl. Opt. 34, 18591865 (1995)

[13]. H. Wang, F. Gan, "High focal depth with a pure-phase apodizer", Appl. Opt. 40, 5658-5662 (2001).

[14].S. Sanyal, A. Ghosh, "High tolerance to spherical aberrations and defects of focus with a birefringent lens", Appl. Opt. 41, 4611-4615 (2002).

[15]. N. George, W. Chi, "Extended depth of field using a logarithmic asphere", J. Opt. A - Pure Appl. Opt. 5, s157-s163 (2003).

[16]. A. Sauceda, J. Ojeda-Castaneda, "High focal depth with fractional-power wave fronts", Opt. Lett. 29, 560-562 (2004).

[17]. A. Castro, J. Ojeda-Castaneda, “Asymmetric phase masks for extended depth of field", Appl. Opt. 43, 3474-3479 (2004).

[18].S. S. Sherif, W. T. Cathey, E. R. Dowski, "Phase plate to extend depth of field of incoherent hybrid imaging system", Appl. Opt. 43, 2709-2721 (2004).

[19]. J. Ojeda-Castaneda, J. E. A. Landgrave, H. M. Escamilla, “Annular phase-only mask for high focal depth", Opt. Lett. 30, 1647-1649 (2005).

[20]. A. Castro, J. Ojeda-Castaneda, A. W. Lohmann, "Bow-tie effect: Differential operator", Appl. Opt. 45, 7878-7884 (2006).

[21]. S. Mezouari, G. Muyo, A. R. Harvey, "Circularly symmetric phase filters for control of primary thirdorder aberrations: coma and astigmatism", J. Opt. Soc. Am. A 23, 1058-1062 (2006).

[22]. G. Mikula, Z. Jaroszewicz, A. Kolodziejczyk, K. Petelczyc, M. Sypek, "Imaging with extended focal depth by means of lenses with radial and angular modulation", Opt. Express 15, 9184-9193 (2007).

[23].M. Somayaji, M. P. Christensen, "Frequency analysis of the wavefront-coding odd-symmetric quadratic phase mask", Appl. Opt. 46, 216-226 (2007).

[24].Y. Takahashi, S. Komatsu, "Optimized free-form phase mask for extension of depth of field in wavefront-coding imaging”, Opt. Lett. 33, 1515-1517 (2008).

[25]. P. Mouroulis, "Depth of field extension with spherical optics”, Opt. Express 16, 12995-13004 (2008).

[26]. N. Caron, Y. Sheng, "Polynomial phase masks for extending the depth of field of a microscope", Appl. Opt. 47, E39-E43 (2008).

[27] Coded aperture imaging, http://astrophysics.gsfc.nasa.gov/cai/

[28].K. Brenner, A. Lohmann, J. Ojeda-Castaneda, "The ambiguity function as a polar display of the OTF", Opt. Commun. 44, 323-326 (1983).

[29] H. Bartelt, J. Ojeda-Castaneda, E. E. Sicre, "Misfocus tolerance seen by simple inspection of the ambiguity function", Appl. Opt. 23, 2693-2696 (1984).

[30]. J. Ojeda-Castañeda, P. Andrés, E. Montes, "Phase-space representation of the Strehl ratio: Ambiguity function", J. Opt. Soc. Am. A 4, 313-317 (1987).

[31]. J. Ojeda-Castaneda, L. R. Berriel-Valdos, E. Montes, “Ambiguity function as a design tool for high focal depth", Appl. Opt. 27, 790-795 (1988).

[32]. R. J. Marks, J. F. Walkup, T. F. Krile, "Ambiguity function display: an improved coherent processor", Appl. Opt. 16, 746-750 (1977).

[33] M. Testorf, B. Hennelly, J. Ojeda-Castañeda, Phase-space Optics: Fundamentals and Applications, McGraw Hill (2010).

[34]. L. W. Alvarez, “Two-element variable-power spherical lens”, U.S. Patent 3,305,294 (3 ${ }^{\text {rd }}$ Dec. 1964). 
[35]. A. W. Lohmann, "Lente focale variabile", Italian Patent 727,848 (19 th June, 1964).

[36]. A. W. Lohmann, "Improvements relating to lenses and to variable optical lens systems formed by such lenses", Patent Specification 998,191, The Patent Office, London (1965).

[37]. A. W. Lohmann, "A new class of varifocal lenses", Appl. Opt. 9, 1669-1671 (1970).

[38]. I. A. Palusinski, J. M. Sasián, J. E. Greivenkamp, "Lateral shift variable aberrations generators", Appl. Opt. 38, 86-90 (1990).

[39]. N. López-Gil, H. C. Howland, B. Howland, N. Charman, R. Applegate, "Generation of third-order spherical and coma aberrations by the use of radially symmetrical fourth-order lenses", J. Opt. Soc. Am. A 15, 2563-2571 (1998).

[40]. J. Ojeda-Castaneda, J. E. A. Landgrave, C. M. Gómez-Sarabia, "The use of conjugate phase plates in the analysis of the frequency response of optical systems designed for an extended depth of field", Appl. Opt. 47, E99-E105 (2008).

[41]. J. Ojeda-Castañeda, C. M. Gómez-Sarabia, "Optical processor arrays for controlling focal length or for tuning the depth of field", Photonics Lett. Poland 3, 44-46 (2011).

[42]. J. Ojeda Castaneda, E. Aguilera Gómez, H. Plascencia Mora, M. Torres Cisneros, E. R. Ledezma Orozco, A. León Martínez, J. S. Pacheco Santamaría, J. G. Martínez Castro, R. C. Salas Segoviano, “Optical system with variable field depth", U. S. Patent 8,159, 573B2 (April 17th, 2012).

[43] J. Ojeda-Castañeda, E. Yépez-Vidal, E. García-Almanza, C. M. Gómez-Sarabia, "Tunable Gaussian mask for extending the depth of field", Photonics Lett. Poland 4, 115-117 (2012)

\section{Introduction}

Pupil masks are useful for shaping the 1-D, point spread function (PSF) of optical spectrometers. The operation is called apodization [1]. The pupil mask reduces the irradiance distribution of the side lobes. And as the secondary effect, the central part of the irradiance PSF is extended. If the irradiance PSF is thought of as the Strehl ratio vs defocus, then apodization increases the depth of focus. Thus, the apodizers used in instrumental spectroscopy are useful for increasing focal depth; provided that they are properly used in 3-D imagery [2].

For extended objects, it is convenient to use the optical transfer function (OTF) for describing methods that reduce the impact of focus errors $[3,4]$. In an early publication we indicated that the depth of field can be extended by the use of an apodizer at the recording step, and a posteriori step of digital restoration [5].

Consequently, optical systems may be considered useful for performing in parallel the two following operations. The optical system should acquire the input irradiance distribution, and at the same time, the optical system should perform an optical pre-processing operation of the input data. The final image is obtained after performing a digital post-processing operation.
Several researches have proposed methods for extending the depth of field of an optical system [2-26]. Here we describe two key concepts that are useful for designing masks that control the depth of field; without modifying the size of pupil aperture.

To our end, we revisited some related publications. However, this paper is not a review paper. Our aim is to link unrelated concepts. We unveil recent results.

The use of the optical system for preprocessing the input, as the system acquires the picture, has been popularized by Dowski and Cathey [12]. They denote the pre-processing operation as "encoding", and the post-processing operation as "decoding". In a way, this notation follows Haeusler proposal, which mimics the usage of the well-known two-step process in optical holography.

Perhaps this is not the best notation. Phase masks that shape the OTF are referred as wavefront coders. Amplitude spatial filters that modify the OTF are in consequence amplitude coders. There are also complex amplitude masks that reduce the spurious oscillations on the OTF, while extending the depth of field. However, the above wording may inspire further research for linking imaging devices with truly coded apertures [27]. 
We note that the present technology allows producing nonconventional masks. And nowadays, it is rather easy to implement fast digital algorithms for post -processing. Consequently, one can foresee that further efforts will be addressed to shape the OTF.

In what follows, we discuss two key concepts that help to shape the OTF for achieving extended depth of field. In section 2, we link anamorphic optical processors with the optical visualization of the optical transfer function (OTF), when focus error is present. In section 3 , we relate the use of Alvarez - Lohmann lenses with adaptable filters that extend the depth of field. In section 4, we summarize our results. For the sake of simplicity, we discuss mainly the 1-D case.

\section{Anamorphic processors and the ambiguity function}

In a previous publication, we established a link between the OTF suffering from focus errors and the ambiguity function [28]. Later on, we explored the use of the ambiguity function for shaping the OTF [29-31]. For the sake of completeness of our presentation, we discuss out-of-focus image formation when using a classical, 4-f, optical processor, as the one in Fig. 1. If the input plane moves away from its initial position, and the detector remains fixed, the optical system suffers focus errors.

Then, the generalized pupil function is

$$
T\left(\mu, W_{2,0}\right)=Q(\mu) \exp \left[i 2 \pi\left(\frac{W_{2,0}}{\lambda}\right)\left(\frac{\mu}{\Omega}\right)^{2}\right] \text {. }
$$

In Eq. (1) we denote as $Q(\mu)$ the complex amplitude transmittance of the pupil mask; $W_{2,0}$ is Hopkins focus error coefficient. The greek

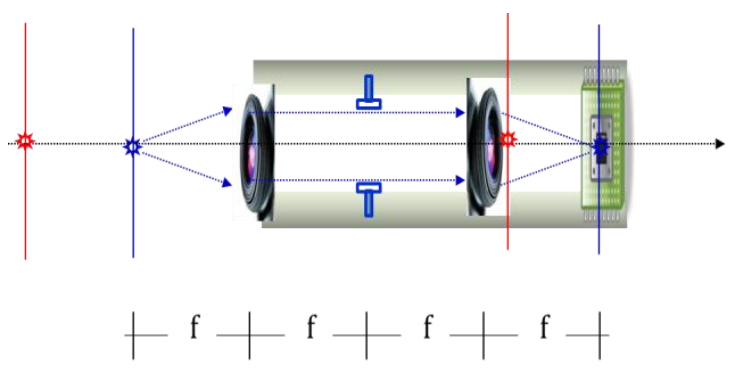

Fig. 1. Schematics of a classical optical processor. The detector plane is at a fixed position. letter $\Omega$ denotes the cut-off spatial frequency of the rectangular pupil aperture. For simplifying the notation we employ the following dimensionless parameter

$$
W=\frac{W_{2,0}}{\lambda} .
$$

It is straightforward to obtain the OTF of Eq. (1), which reads

$$
\begin{array}{r}
H_{Q}(\mu, W)=N \int_{-\infty}^{+\infty} Q\left(v+\frac{\mu}{2}\right) Q^{*}\left(v-\frac{\mu}{2}\right) \times \\
\quad \times \exp \left[i 2 \pi\left(\frac{2 W \mu}{\Omega^{2}}\right) v\right] d v .
\end{array}
$$

In Eq. (3) the upper case letter $N$ denotes a suitable normalization factor. On the other hand, the ambiguity function of the pupil mask, $Q(\mu)$, is

$$
\begin{array}{r}
A_{Q}(\mu, y)=N \int_{-\infty}^{+\infty} Q\left(v+\frac{\mu}{2}\right) Q^{*}\left(v-\frac{\mu}{2}\right) \times \\
\times \exp [i 2 \pi y v] d v
\end{array}
$$

From a simple comparison between Eqs. (3) and (4), we note that both expressions are interrelated if

$$
y=\left(\frac{2 W}{\Omega^{2}}\right) \mu .
$$

Then, the variations of the OTF with variable focus errors can be visualized from a display of the ambiguity function, as in Fig. 2.

If we trace a straight-line from $(0,0)$ to $(\mu, y)$, the slope is proportional to the amount of focus error, as indicated in Eq. (5). For a fixed value of $W$, say $W=W_{0}$, the values of the ambiguity function (straight-line with constant slope) are the values of the OTF $H_{Q}\left(\mu, W_{0}\right)$.

If we trace a vertical line, along the $y$-axis, for a fixed value of the spatial frequency, say $\mu=\sigma$, then we obtain the values of the OTF for variable W. That is, $H_{Q}(\sigma, W)$.

Next, we recognize that a display of the ambiguity function, as that in Fig. 2, can be obtained by implementing the anamorphic processor that is depicted in Fig. 3. See reference [32,33].

The input for the anamorphic processor is the "Product Spectrum" 


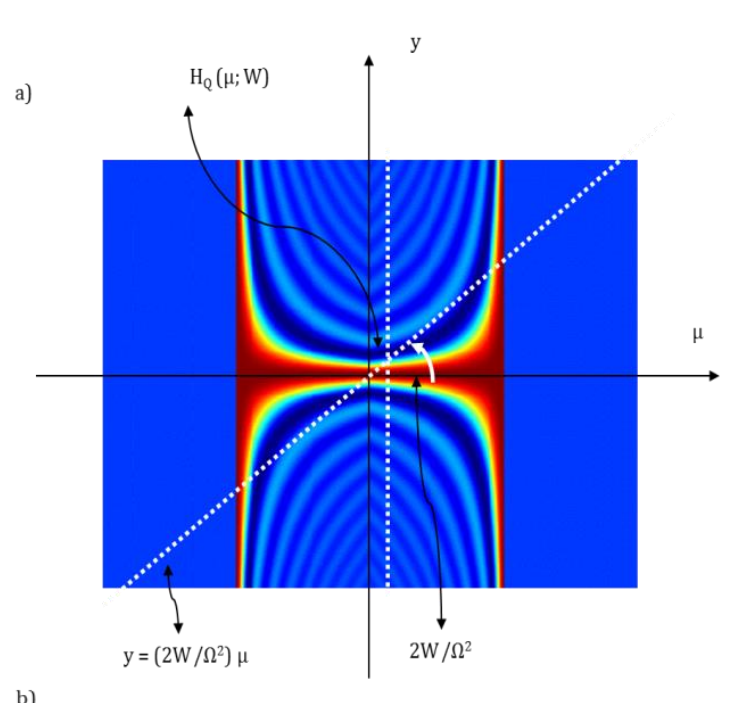

b)

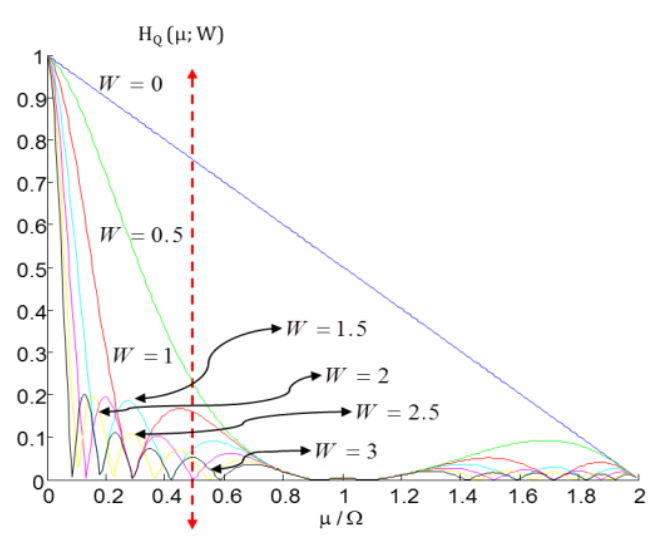

Fig. 2. Fig.2. Display of the values of OFT using the ambiguity function: a) ambiguity function display, b) traditional display for the following values $W=0,0.5,1,1.5,2,2.5$ and 3 .

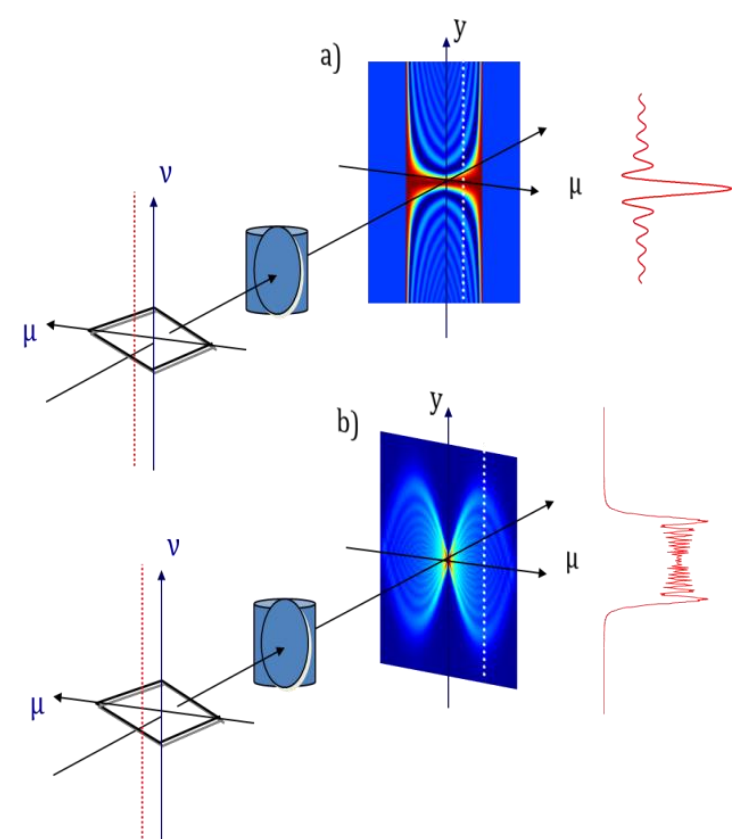

Fig. 3. Anamorphic optical processor for generating the ambiguity function.

$$
A_{Q}(\mu, v)=Q\left(v+\frac{\mu}{2}\right) Q^{*}\left(v-\frac{\mu}{2}\right)
$$

In Fig. 3, the anamorphic processor performs an imaging operation, with unit magnification, along the horizontal axis. And the processor implements a Fourier transform along the vertical axis.

For a clear pupil aperture, a vertical line along the ambiguity function comes from the Fraunhofer diffraction pattern of a vertical line along $v$-axis, which is a rectangular window with variable width. That is,

$$
P_{Q}(\mu, v)=\operatorname{rect}\left(\frac{v}{2 \Omega-|\mu|}\right) \operatorname{rect}\left(\frac{\mu}{4 \Omega}\right) \text {. }
$$

In Eq. (7) we use the common, generic notation $\operatorname{rect}(x / X)$ for representing a window function that is equal to unity inside the interval $-X / 2 \leq x \leq X / 2$; and it is equal to zero elsewhere. Then, as depicted in Fig. 3(a), the ambiguity function is composed of a series of sinc functions

$$
\begin{aligned}
A_{Q}(\mu, y)=N & \int_{-\infty}^{+\infty} P_{Q}(\mu, v) e^{i 2 \pi y v} d v= \\
& =\left(1-\frac{|\mu|}{2 \Omega}\right) \operatorname{sinc}\left[\left(1-\frac{|\mu|}{2 \Omega}\right) y\right] .
\end{aligned}
$$

Next, we consider another possibility. If at the "Product spectrum" we have a vertical line that has a quadratic phase factor,

$$
\begin{aligned}
P_{Q}(\mu, v) & = \\
= & e^{i 2 \pi a\left(\frac{v}{\Omega}\right)^{2}} \operatorname{rect}\left(\frac{v}{2 \Omega-|\mu|}\right) \operatorname{rect}\left(\frac{\mu}{4 \Omega}\right) .
\end{aligned}
$$

For obtaining this type of "Product Spectrum", one needs a cubic phase mask, as proposed by Dowski and Cathey [12]. By taking the Fourier transform of Eq. (9), along the $v$ variable, we obtain

$$
\begin{aligned}
& A_{Q}(\mu, y)= \\
& \quad=\operatorname{rect}\left(\frac{\mu}{4 \Omega}\right) \int_{-\frac{2 \Omega-|\mu|}{2}}^{\frac{2 \Omega-|\mu|}{2}} e^{i 2 \pi a\left(\frac{v}{\Omega}\right)^{2}} e^{i 2 \pi y v} d \nu .
\end{aligned}
$$

It is apparent from Eq. (10) that along the $y$-axis, the ambiguity function is composed of a series of Fresnel diffraction patterns of rectangular windows, which have variable width. See Fig. 
3(b). Since any vertical line is a Fresnel diffraction pattern, then the ambiguity function spreads all over the $(\mu, y)$ plane. This behaviour is known as the "bow-tie effect" [20]. As a consequence, the OTF's have low sensitivity to focus errors.

One can extrapolate the previous result, by introducing high order aberration polynomials, as phase variations at the "Product Spectrum". That is,

$$
\begin{aligned}
& P_{Q}(\mu, v)= \\
& \quad=e^{i 2 \pi a\left(\frac{v}{\Omega}\right)^{2 n}} \operatorname{rect}\left(\frac{v}{2 \Omega-|\mu|}\right) \operatorname{rect}\left(\frac{\mu}{4 \Omega}\right) .
\end{aligned}
$$

with $n=1,2,3, \ldots$ This type of "Product Spectrum" may be obtained by using a high order phase mask,

$$
Q(\mu)=e^{i 2 \pi a\left(\frac{\nu}{\Omega}\right)^{2 n+1}} \operatorname{rect}\left(\frac{\mu}{2 \Omega}\right) .
$$

The phase masks in Eq. (12) spread the ambiguity function along the vertical axis, and consequently the masks is able to extend the depth of field. However, these types of masks (including the cubic phase mask) introduce spurious oscillations. For reducing this undesirable feature, one can use a Gaussian apodizer; as part of the spatial filter, as is schematically depicted in Fig. 4.

Hence, for extending the depth of field, this mask has the following complex amplitude transmittance

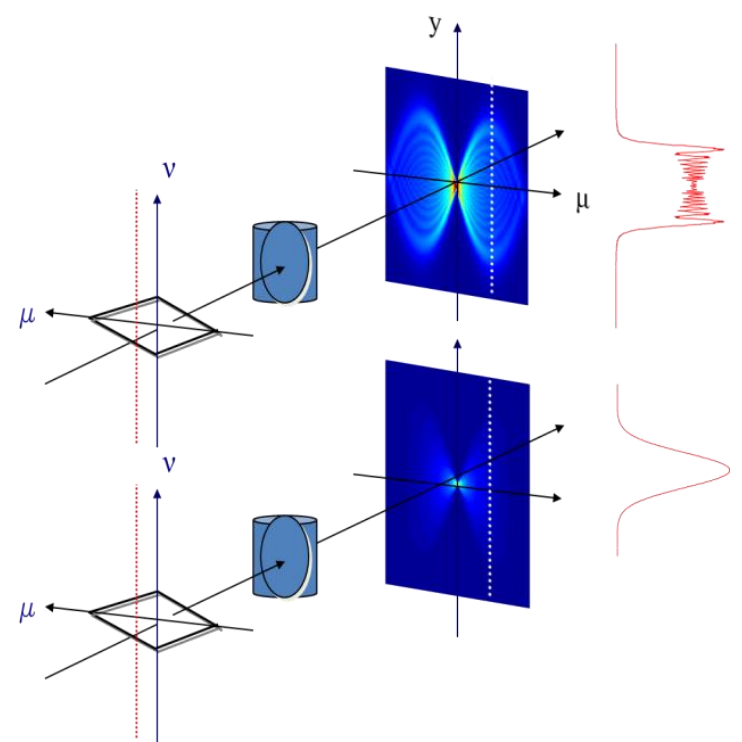

Fig. 4. Schematics of an anamorphic processor with a Gaussian apodizer.

$$
Q(\mu)=e^{i 2 \pi a\left(\frac{v}{\Omega}\right)^{2 n+1}} e^{-2 \pi c\left(\frac{\mu}{\Omega}\right)^{2}} \operatorname{rect}\left(\frac{\mu}{2 \Omega}\right) .
$$

In Eq. (13) the lower case letter $c$ denotes a damping factor, in the amplitude mask with Gaussian profile. See reference [10].

In Fig. 5(a), we show the modulus of the OTF (the Modulation Transfer Function, MTF) of the cubic phase mask for $W=0$ and for $W=3$. One note that there are spurious oscillations, as one should expect from a Fresnel diffraction pattern.

In Fig. 5(b), we show the MTF of the masks in Eq. (13). We note that unwanted oscillations are reduced, for both cases $W=0$ and $W=3$.

In Fig. 6 we summarize, in a graphical manner, the previous results. From left to right, we depict the modulus of the ambiguity function for the clear aperture. Next, we show the modulus of the ambiguity function for the cubic phase masks. And finally, we depict the modulus of the ambiguity function for the masks in Eq. (13). It is clear from Fig. 6 that the complex amplitude mask generates an extended ambiguity function, with damped variations along the vertical axis. If in Eq. (13) we select the values $a=15$ and $c=0.7$, then we obtain the MTFs depicted in Fig. 7. These MTFs vary slowly with focus error.

In the following section, we describe a method for implementing tunable versions of

a)

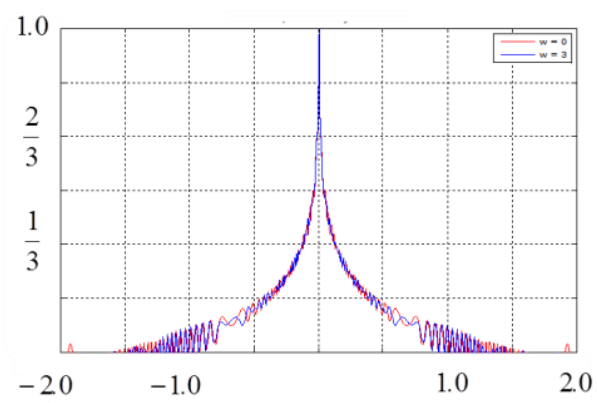

b)

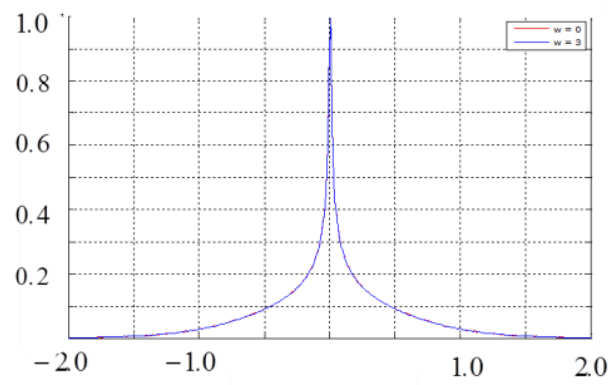

Fig. 5. Spurious oscillations on the MTF: a) cubic phase mask alone, b) cubic phase mask with a Gaussian apodizer. 

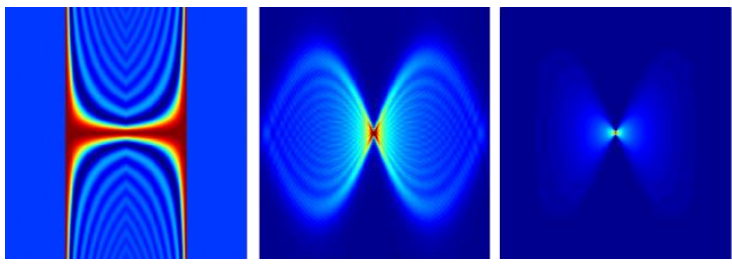

Fig. 6 Modulus of the Ambiguity function: a) rectangular pupil aperture, b) the bow-tie effect, c) Gaussian apodized.
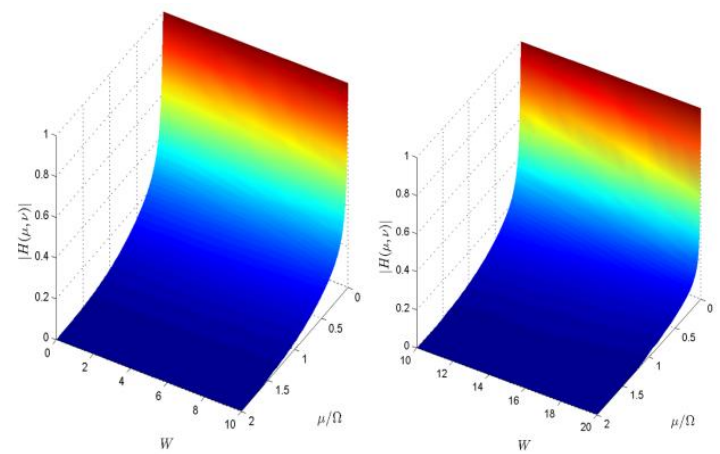

Fig. 7. MTF vs focus error $|H(\mu ; W)|$ for two different ranges of $W$.

the cubic phase mask, as well as tunable version of the Gaussian apodizer.

\section{Adaptable spatial filters}

Almost at the same time, Alvarez and Lohmann proposed similar methods for implementing a varifocal lens [34-37]. Their method employs two phase masks that form a pair. The amplitude transmittance of a single mask is the complex conjugate of the other mask. Then, by introducing a lateral displacement between the two masks, say $\sigma$, one sets a varifocal lens. For further examples see reference [38].

Our following discussion extends the results reported in references [38-43]. If one neglects the size of the mask, one element (of the AlvarezLohmann pair) has the following complex amplitude transmittance

$$
Q(\mu)=e^{i 2 \pi a\left(\frac{\nu}{\Omega}\right)^{3}}
$$

In Eq. (14) the lower case letter $a$ denotes the optical path difference in units of the wavelength $\lambda$. If a lateral displacement is introduced, between the elements of the pair, one sets the following generalized pupil function representing a lens

$$
\begin{aligned}
& P_{Q}(v, \sigma)=Q\left(v+\frac{\sigma}{2}\right) Q^{*}\left(v-\frac{\sigma}{2}\right)= \\
& \quad=e^{i\left(\frac{a \pi}{2}\right)\left(\frac{\sigma}{\Omega}\right)^{3}} e^{i 2 \pi\left(\frac{a \sigma}{\Omega}\right)\left(\frac{v}{\Omega}\right)^{2}} .
\end{aligned}
$$

In Eq. (15) the quadratic variation in $v$ represents a lens. By changing $\sigma$, one can tune the power of a lens. The result in Eq. (15) is similar to the result in Eq. (9). Hence, we claim the following. By visualizing the amplitude PSF of the pair, one visualizes the ambiguity function of a single element of the pair. That is,

$$
\begin{array}{rl}
p_{Q}(x, \sigma)=N \int_{-\infty}^{+\infty} P_{Q}(v, \sigma) e^{i 2 \pi x v} & d v= \\
=A_{Q}(\sigma, x) .
\end{array}
$$

This is remarkable result. Now, we have a physical method (the use of a pair of phase masks) for understanding the influence that a single element has on the behaviour of the ambiguity function.

By applying this result, one can propose the use of a mask that has phase variations to the four-power. This mask generates a cubic phase mask, with controllable optical path difference [37-40]. In mathematical terms, the complex amplitude transmittance of a single element is

$$
Q(v)=e^{i 2 \pi a\left[\left(\frac{v}{\Omega}\right)^{4}-\frac{1}{2}\left(\frac{v}{\Omega}\right)^{2}\right]}
$$

If we employ a pair, and we introduce a lateral displacement between the members of the pair, we generates the following generalized pupil function

$$
\begin{aligned}
P_{Q}(v, \sigma) & =Q\left(v+\frac{\sigma}{2}\right) Q^{*}\left(v-\frac{\sigma}{2}\right)= \\
& =e^{i 2 \pi\left(\frac{4 a \sigma}{2}\right)\left(\frac{v}{\Omega}\right)^{3}} e^{i 2 \pi\left(\frac{a \sigma}{\Omega}\right)\left[\left(\frac{\sigma}{\Omega}\right)^{2}-1\right]\left(\frac{v}{\Omega}\right)} .
\end{aligned}
$$

It is clear from Eq. (18) that by changing the value of $\sigma$, we can change the optical path difference of a cubic phase mask. However, the proposed phase mask generates a linear phase variation, which has the following tilt coefficient

$$
C_{1}=\left(\frac{a \sigma}{\Omega}\right)\left[\left(\frac{\sigma}{\Omega}\right)^{2}-1\right] \text {. }
$$

The influence of this unwanted term can be reduced by properly selecting the values of the initial optical path difference (parameter $a$ ) and the maximum value of the lateral displacement $\sigma$. 
If one uses linear regression and a Euclidean distance, as in ref. [10], it is possible to identify that a good value for the maximum optical path difference, of the cubic phase, is

$$
\left(\frac{4 a \sigma}{\Omega}\right)_{\max }=16
$$

Hence, the maximum value for the lateral displacement is

$$
\sigma_{\max }=4 \frac{\Omega}{a} .
$$

By using Eqs. (20) and (21) in Eq. (19), we obtain that the tilt coefficient can be expressed as

$$
C_{1}=\left(\frac{4 \sigma}{\sigma_{\max }}\right)\left[\left(\frac{4 \sigma}{a \sigma_{\max }}\right)^{2}-1\right] .
$$

From Eq. (22) we claim that a good choice for the initial optical path difference is $a=4$. Therefore, the tilt coefficient reads

$$
C_{1}=\left(\frac{4 \sigma}{\sigma_{\max }}\right)\left[\left(\frac{\sigma}{\sigma_{\max }}\right)^{2}-1\right] .
$$

It is apparent form Eq. (23) that now the tilt coefficient has zero value at $\sigma=0$, and at $\sigma=\sigma_{\max }$.

Next, we describe the use of a pair of amplitude masks for setting a Gaussian spatial filter, with adjustable width. We start our discussion, by considering the amplitude transmittance of the first member of the proposed pair

$$
T_{1}(\mu)=e^{-c\left[1+\left(\frac{\mu}{2 \Omega}\right)^{3}\right]} \operatorname{rect}\left(\frac{\mu}{4 \Omega}\right) .
$$

As before, in Eq. (24) we use a lower case letter $c$ for denoting a dimensionless damping factor of the Gaussian function. From Eq. (24), we note that the length of the mask is $4 \Omega$. Furthermore, $T_{1}(-2 \Omega)=1, \quad$ while $\quad T_{1}(2 \Omega)=e^{-2 a} . \quad$ The amplitude transmittance of the of the second mask is

$$
T_{2}(\mu)=e^{-c\left[1-\left(\frac{\mu}{2 \Omega}\right)^{3}\right]} \operatorname{rect}\left(\frac{\mu}{4 \Omega}\right) .
$$

Again we recognize that in Eq. (25) the length of the mask is $4 \Omega$, and that $T_{2}(-2 \Omega)=e^{-2 a}$, while $T_{2}(2 \Omega)=1$. In Fig. 8 we depict schematically the two masks on top of the pupil aperture, which has a length equal to $2 \Omega$.
Next, we introduce a lateral displacement, $2 \sigma$; between the masks. The parameter $\sigma$ varies from zero to $\Omega$. Hence, Fig. 8 depicts the two extreme cases. Next, we recognize that the overall amplitude transmittance inside the pupil aperture is

$$
T(\mu, \sigma)=T_{1}(\mu+\sigma) T_{2}(\mu-\sigma) \operatorname{rect}\left(\frac{\mu}{2 \Omega}\right) .
$$

By substituting Eqs. (24) and (25) in Eq. (26) we obtain

$$
\begin{aligned}
T(\mu, \sigma) & = \\
& =e^{-2 c\left[1+\left(\frac{\sigma}{2 \Omega}\right)^{3}\right]} e^{-\left(\frac{3 c \sigma}{4 \Omega}\right)\left(\frac{\mu}{\Omega}\right)^{2}} \operatorname{rect}\left(\frac{\mu}{2 \Omega}\right) .
\end{aligned}
$$

It is apparent from Eq. (27) that inside the pupil aperture, the overall amplitude transmittance varies as a Gaussian function. In Fig. 9(a), we depict the case $\sigma=0$. In this case, we obtain a uniform attenuating window. In Fig. 9(b), we depict the amplitude transmittances associated to the case $\sigma>0$. We note that for $\sigma \neq 0$, it is relevant to identify the parameter $\mu_{0}$, which known as the half width at half maximum.

$$
\mu_{0}=2 \Omega \sqrt{\frac{\ln (2)}{3 c(\sigma / \Omega)}} .
$$

Here, we assume that the maximum displacement is $\sigma=\Omega$. Then by using Eq. (28) we

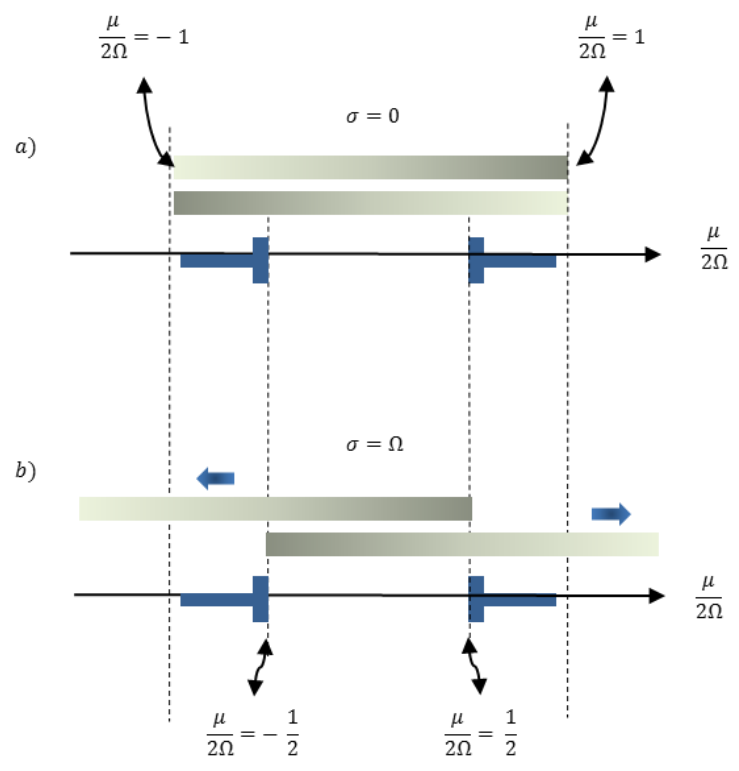

Fig. 8. Schematics of the two amplitude masks over the pupil aperture. 

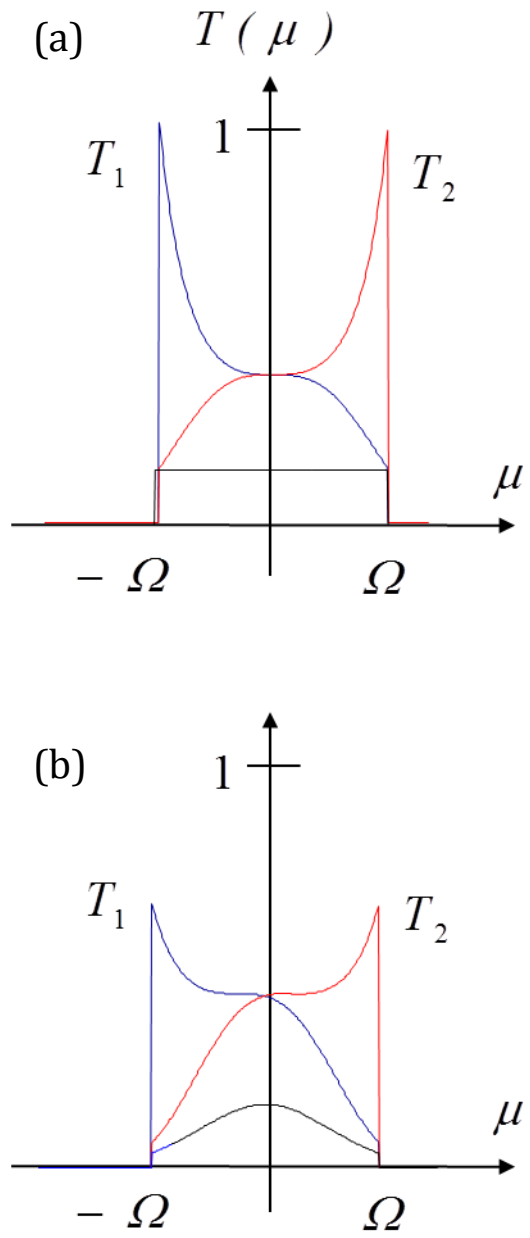

Fig. 9. Overall amplitude transmittance: (a) for $\sigma=0$, (b) for $\sigma>0$.

obtain the mathematical expression for setting the damping factor

$$
c=\frac{\ln (2)}{3\left(\mu_{0} / \Omega\right)^{2}} .
$$

We note that Eq. (29) is useful for setting the value of the dimensionless damping factor, for a given value of half width at half maximum. For example, if the half width at half maximum is $\mu_{0}=\Omega / \sqrt{2}$, then $c=8 \ln (2) / 3$. Next, we report the 2-D version of the proposed filter.

The amplitude transmittance of the first mask is

$T_{1}(\mu, v)=e^{-c\left[1+\left(\frac{\mu}{2 \Omega}\right)^{3}+3\left(\frac{v}{2 \Omega}\right)^{2}\left(\frac{\mu}{2 \Omega}\right)\right]} \operatorname{rect}\left(\frac{\mu}{4 \Omega}\right)$.

The amplitude transmittance of the second mask is

$$
T_{2}(\mu, v)=e^{-c\left[1+\left(\frac{\mu}{2 \Omega}\right)^{3}-3\left(\frac{v}{2 \Omega}\right)^{2}\left(\frac{\mu}{2 \Omega}\right)\right]} \operatorname{rect}\left(\frac{\mu}{4 \Omega}\right) .
$$

At the corners of the rectangular window, the 2D masks have either maximum transmittance (equal to unity) or minimum transmittance (equal to $e^{-8 a}$ ). The amplitude transmittance of the proposed, 2-D filter is

$$
\begin{aligned}
T(\mu, v, \sigma)=T_{1}(\mu+\sigma, v) T_{2}(\mu-\sigma, v) \times & \\
& \times \operatorname{rect}\left(\frac{\mu}{2 \Omega}\right) \operatorname{rect}\left(\frac{v}{2 \Omega}\right) .
\end{aligned}
$$

By substituting Eqs. (30) and (31) in Eq. (32) we obtain

$$
\begin{array}{r}
T(\mu, v, \sigma)=e^{-c\left[1+\left(\frac{\sigma}{2 \Omega}\right)^{3}\right]} e^{-\left(\frac{3 c \sigma}{4 \Omega}\right)\left[\left(\frac{\mu}{\Omega}\right)^{2}+\left(\frac{v}{\Omega}\right)^{2}\right]} \times \\
\times \operatorname{rect}\left(\frac{\mu}{2 \Omega}\right) \operatorname{rect}\left(\frac{v}{2 \Omega}\right) .
\end{array}
$$

We note from Eq. (33) that indeed the overall amplitude transmittance varies, with $\mu$ and $v$, as a 2-D, Gaussian function inside a rectangular window. In Fig. 10, we show the contour levels of Eq. (33) for three values of $\sigma$.

Finally, we recognize that if one uses simultaneously the tunable cubic phase mask and the tunable Gaussian amplitude mask, then one has two extra degrees of freedom for setting the pre-processing filter. If one uses linear regression and a Euclidean distance, one can identify good values for the optical path difference, $a$, and for the damping factor $c$.

In Fig. 11 we show a set of pictures that display the ambiguity function, as one changes either the value of the optical path difference (columns of the matrix of pictures)or the value of the damping factor (rows of the matrix of pictures). The minimum Euclidean distance is around $a=16$ and $c=0.7$, which is the picture at the last column, last row.

\section{Conclusions}

Summarizing, we have discussed two key concepts that are useful for designing phase masks that extend the depth of field. The first key concept links the use of anamorphic processors for visualizing the ambiguity function with the out-of-focus OTFs. We have indicated the convenience of generating high order 

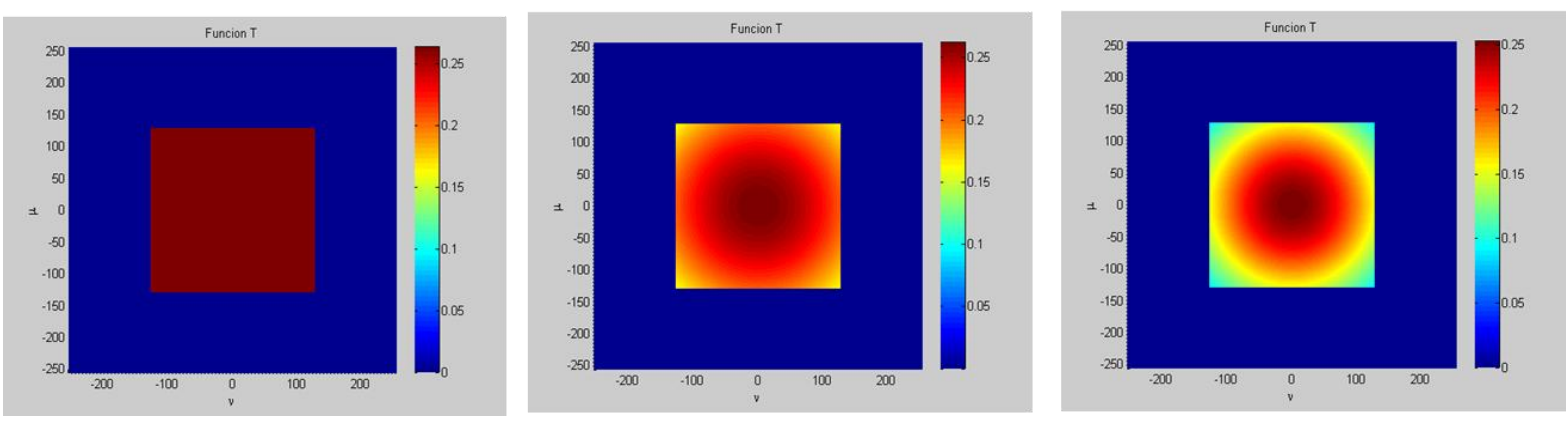

Fig. 10. Contour levels of the overall amplitude transmittance for three values of $\sigma$.
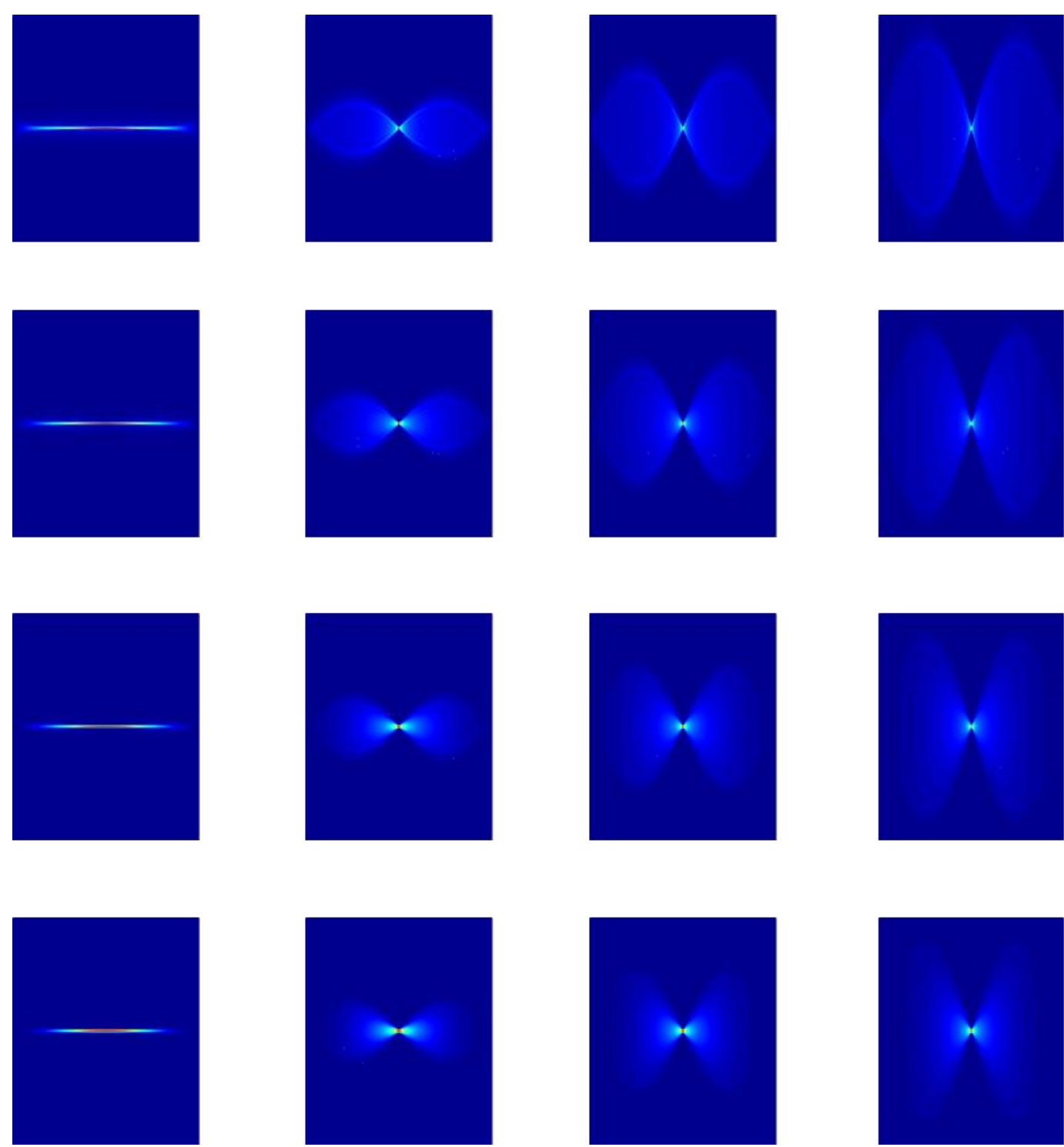

Fig. 11. Display of ambiguity functions for variable optical path difference and variable damping factor. 
diffraction patterns, when visualizing the ambiguity function. We have noted that for evaluating the ambiguity function, the integrand in the Fourier transform resemblance the physical arrangement of the Alvarez-Lohmann phase pair. We have recognized that this analogy is useful for proposing an adjustable phase mask, which controls the depth of field without changing the size of the pupil aperture. Furthermore, since in general the type of phase masks discussed generated spurious oscillation in the MTF, we have proposed a method that reduces these unwanted oscillations. We unveil the use of two amplitude masks for generating a
Gaussian attenuation filter, with tunable halfwidth. We have reported the analytical expressions for these type of filters in 1-D and in 2-D. The later method is an extension of the Alvarez-Lohmann lenses, but for amplitude mask.

\section{Acknowledgements}

This paper is presented in memory of Professor Carlos Gómez-Reino. We are indebted to CoNaCyT for the research grant 157673 and to PROMEP for the grants 103.5/10/4612 and PTC197 D. We extend our gratitude to the reviewers for their useful suggestions. 